\title{
Дифференциальная туннельная проводимость в многокомпонентных твердых растворах $\mathrm{Bi}_{2-x} \mathrm{Sb}_{x} \mathrm{Te}_{3-y-z} \mathrm{Se}_{y} \mathrm{~S}_{z}$
}

\author{
(С) Л.Н. Лукьянова, И.В. Макаренко, О.А. Усов \\ Физико-технический институт им. А.Ф. Иофре Российской академии наук, \\ 194021 Санкт-Петербург, Россия \\ E-mail: lidia.lukyanova@mail.ioffe.ru \\ Поступила в Редакцию 12 августа 2021 г. \\ В окончательной редакции 28 августа 2021 г. \\ Принята к публикации 28 августа 2021 г.
}

\begin{abstract}
Дифференциальная туннельная проводимость межслоевой поверхности Ван-дер-Ваальса (0001) исследована в многокомпонентных твердых растворах $n$ - $\mathrm{Bi}_{2-x} \mathrm{Sb}_{x} \mathrm{Te}_{3-y-z} \mathrm{Se}_{y} \mathrm{~S}_{z}$ при $x=0.2, y=z=0.09$, оптимизированных для температур вблизи комнатной с высоким фактором мощности, и при $x=0.4, y=0, z=0.06 \mathrm{c}$ оптимальными термоэлектрическими свойствами для низких температур и высоким коэффициентом Зеебека. Показано, что интенсивность флуктуаций энергии точки Дирака $\Delta E_{\mathrm{D}}$, смещение потолка валентной зоны и наличие поверхностных уровней в запрещенной зоне, образованных примесными дефектами, определяются составом и термоэлектрическими свойствами твердых растворов. Вклад поверхностных состояний фермионов Дирака возрастает в твердом растворе $n$ - $\mathrm{Bi}_{1.8} \mathrm{Sb}_{0.2} \mathrm{Te}_{2.82} \mathrm{Se}_{0.09} \mathrm{~S}_{0.09}$ с высоким параметром мощности за счет значительного уменьшения поверхностной концентрации вблизи точки зарядовой нейтральности и роста подвижности фермионов.
\end{abstract}

Ключевые слова: теллурид висмута, твердые растворы, топологический изолятор, дифференциальная туннельная проводимость, коэффициент Зеебека.

DOI: $10.21883 /$ FTP.2021.12.51694.20

\section{1. Введение}

Слоистые халькогениды висмута и сурьмы являются широко известными термоэлектриками и перспективными трехмерными топологическими изоляторами (ТИ) с аномальными электронными свойствами поверхностных состояний фермионов Дирака [1], которые определяют возможности использования рассматриваемых слоистых материалов в различных областях физики в качестве фотодетекторов [2], полевых транзисторов [3], в спинтронике, [4], в лазерах [5] и термоэлектричестве [6,7].

Одним из методов исследований характеристик поверхностных электронных состояний фермионов Дирака является метод сканирующей туннельной спектроскопии (СТC), определяющий локальную дифференциальную туннельную проводимостиь $d I_{t} / d U$, пропорциональную электронной плотности состояний [8].

Настоящая работа посвящена исследованию топологических поверхностных состояний фермионов Дирака методом (СТC) в слоистых многокомпонентных твердых растворах $n$-типа $(\mathrm{Bi}, \mathrm{Sb})_{2}(\mathrm{Te}, \mathrm{Se}, \mathrm{S})_{3}$ с оптимальными термоэлектрическими свойствами для области температур ниже и выше комнатной. Рассматриваются особенности туннельных спектров, связанные со сдвигом положения точки Дирака относительно уровня Ферми, флуктуации энергии точки Дирака $\Delta E_{\mathrm{D}}$, образование примесных уровней в запрещенной зоне, а также изменения поверхностной концентрации фермионов Дирака в зависимости от величины коэффициента Зеебека, фактора мощности и подвижности носителей заряда в твердых растворах при различных замещениях атомов в подрешетках $\mathrm{Bi}$ и Те.

\section{2. Сканирующая туннельная спектроскопия. Энергия точки Дирака}

Исследования топологических поверхностных состояний фермионов Дирака в многокомпонентных твердых растворах $n$ - $\mathrm{Bi}_{1.6} \mathrm{Sb}_{0.4} \mathrm{Te}_{2.94} \mathrm{Se}_{0.06} \quad$ и $n-\mathrm{Bi}_{1.8} \mathrm{Sb}_{0.2} \mathrm{Te}_{2.82} \mathrm{Se}_{0.09} \mathrm{~S}_{0.09}$ с различным замещением атомов в подрешетках $\mathrm{Bi}$ и Те проводили с помощью анализа спектров дифференциальной туннельной проводимости $d I_{t} / d U$, измеренных в зависимости от напряжения $U$ методом СТС при комнатной температуре.

Рассматриваемые материалы представляют собой анизотропные слоистые пятислойные структуры, разделенные щелями Ван-дер-Ваальса вдоль межслоевых плоскостей (0001). Образцы твердых растворов для измерений были вырезаны из монокристаллических зерен объемных слитков, выращенных методом направленной кристаллизации. Монокристаллические зерна разрезали вдоль плоскостей (0001), ориентированных по оси роста слитка, перпендикулярной кристаллографической оси $c$. Термоэлектрические характеристики объемных образцов представлены в табл. 1 .

На рис. 1 приведены спектры дифференциальной туннельной проводимости $d I_{t} / d U$, измеренные в нескольких произвольно выбранных точках на поверхности (0001), и 
Таблица 1. Коэффициент Зеебека $S$, фактор мощности $S^{2} \sigma$, где $\sigma$ - удельная электропроводность и подвижность $\mu_{0}$ с учетом вырождения носителей заряда при $T=300 \mathrm{~K}$

\begin{tabular}{c|c|c|c|c}
\hline № п. п. & Состав & $\begin{array}{c}S, \\
\mathrm{MKB} \cdot \mathrm{K}^{-1}\end{array}$ & $\begin{array}{c}S^{2} \sigma, \\
10^{-6} \mathrm{BT} \cdot \mathrm{cm}^{-1} \cdot \mathrm{K}^{-2}\end{array}$ & $\begin{array}{c}\mu_{0}, \\
\mathrm{~cm}^{2} \cdot \mathrm{B}^{-1} \cdot \mathrm{c}^{-1}\end{array}$ \\
\hline 1 & $n-\mathrm{Bi}_{1.6} \mathrm{Sb}_{0.4} \mathrm{Te}_{2.94} \mathrm{Se}_{0.06}$ & -234 & 42.6 & $250[9]$ \\
2 & $n-\mathrm{Bi}_{1.8} \mathrm{Sb}_{0.2} \mathrm{Te}_{2.82} \mathrm{Se}_{0.09} \mathrm{~S}_{0.09}$ & -280 & 29.4 & $350[9]$
\end{tabular}

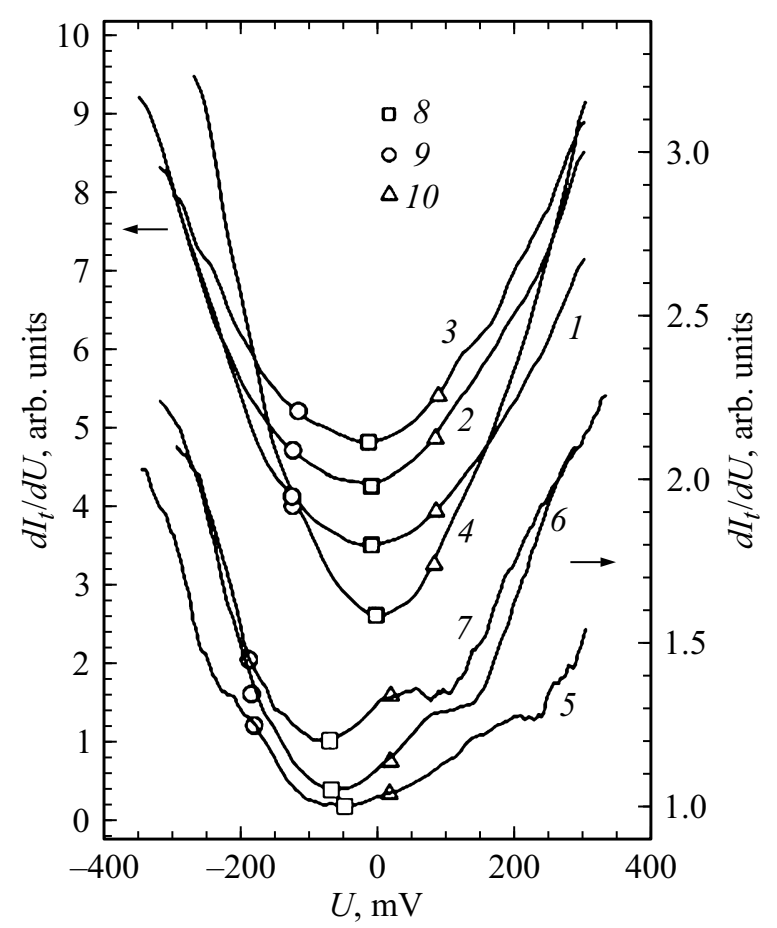

Рис. 1. Нормированная дифференциальная туннельная проводимость $d I_{t} / d U$ в зависимости от напряжения $U$ в твердых растворах $n$ - $\mathrm{Bi}_{1.8} \mathrm{Sb}_{0.2} \mathrm{Te}_{2.82} \mathrm{Se}_{0.09} \mathrm{~S}_{0.09}(1-4)$ и $n$ - $\mathrm{Bi}_{1.6} \mathrm{Sb}_{0.4} \mathrm{Te}_{2.94} \mathrm{Se}_{0.06}(5-7) .8$ - точка Дирака $E_{\mathrm{D}}, 9-$ положение краев валентной зоны $E_{v}$ и $10-$ зоны проводимости $E_{c}$. Кривые 1-7 произвольно смещены вверх для наглядности.

показано положение точки Дирака $E_{\mathrm{D}}$, которое соответствует минимуму на зависимости $d I_{t} / d U$ от $U$ в твердых растворах $n$-типа. Энергия точки $E_{\mathrm{D}}$ изменяется в зависимости от состава твердого раствора и термоэлектрических параметров. В составе $n-\mathrm{Bi}_{1.8} \mathrm{Sb}_{0.2} \mathrm{Te}_{2.82} \mathrm{Se}_{0.09} \mathrm{~S}_{0.09}$ с высоким фактором мощности (табл. 1, рис. 1, кривые $1-4)$ уровень Ферми находится поблизости от точки Дирака $E_{\mathrm{D}}$, расположенной в запрещенной зоне в окрестности точки зарядовой нейтральности топологических поверхностных фермионов, для которых, в соответствии c $[10,11]$, характерно аномальное повышение скорости и подвижности фермионов.

Аналогичное положение точки Дирака $E_{\mathrm{D}}$, вблизи уровня Ферми, наблюдалось также в твердом растворе $n-\mathrm{Bi}_{2} \mathrm{Te}_{2.94} \mathrm{~S}_{0.06}$ с замещениями $\mathrm{Te} \rightarrow \mathrm{S}$ [12], оптимизированном для комнатной температуры при близких тер- моэлектрических параметрах при $S=-220$ мкВ $\cdot \mathrm{K}^{-1}$ и $S^{2} \sigma=43.4 \cdot 10^{-6} \mathrm{BT} \cdot \mathrm{cm}^{-1} \cdot \mathrm{K}^{-2}$, что и в многокомпонентном твердом растворе (рис. 1, кривые 1,2 ).

\section{3. Флуктуации точки Дирака}

Измеренная в четырех точках поверхности $(0001)$ энергия $E_{\mathrm{D}}$ в твердом растворе $n-\mathrm{Bi}_{1.8} \mathrm{Sb}_{0.2} \mathrm{Te}_{2.82} \mathrm{Se}_{0.09} \mathrm{~S}_{0.09}, \quad$ позволяет оценить флуктуации $\Delta E_{\mathrm{D}}$ по отношению к среднему значению $\left\langle E_{\mathrm{D}}\right\rangle$ из отношений $\Delta E_{\mathrm{D}} /\left\langle E_{\mathrm{D}}\right\rangle$. Относительные флуктуации, связанные с изменением плотности состояний в $n-\mathrm{Bi}_{1.8} \mathrm{Sb}_{0.2} \mathrm{Te}_{2.82} \mathrm{Se}_{0.09} \mathrm{~S}_{0.09}$, варьируются от 65 до $2 \%$ в различных точках межслоевой поверхности (0001) (рис. 1, кривые 1,2). В твердом растворе $n-\mathrm{Bi}_{1.6} \mathrm{Sb}_{0.4} \mathrm{Te}_{2.94} \mathrm{Se}_{0.06}$ (рис. 1, кривые 5-7), оптимизированном для температур ниже комнатной, при высокой величине $S=-280 \mathrm{M \kappa B} \cdot \mathrm{K}^{-1}$ энергия $E_{\mathrm{D}}$ была существенно больше, чем в $n-\mathrm{Bi}_{1.8} \mathrm{Sb}_{0.2} \mathrm{Te}_{2.82} \mathrm{Se}_{0.09} \mathrm{~S}_{0.09}$, а флуктуации $\Delta E_{\mathrm{D}}$ уменьшались от 23 до $8 \%$ (кривые 1,2 ).

Положение краев валентной зоны $E_{v}$ и зоны проводимости $E_{c}$ в исследуемых твердых растворах определяли методом нормализованной дифференциальной проводимости из точек перегиба кривой $\left(d I_{t} / d U\right) /\left(\left|I_{t}\right|(U)\right)$. Такая методика достаточно точно воспроизводит особенности дифференциальной туннельной проводимости при малых туннельных токах $[12,13]$. Однако для уточнения положения $E_{v}$ и $E_{c}$ в составе $n-\mathrm{Bi}_{1.8} \mathrm{Sb}_{0.2} \mathrm{Te}_{2.82} \mathrm{Se}_{0.09} \mathrm{~S}_{0.09}$ дополнительно использовали расчет второй производной $d I_{t}^{2} / d U^{2}[8]$.

Энергия краев зон $E_{v}$ и $E_{c}$ (рис. 1) определяется составом твердого раствора и величинами коэффициента Зеебека и фактора мощности (табл. 1).

Положение края валентной зоны $E_{v}$, определенное для различных фрагментов поверхности, в составе $n-\mathrm{Bi}_{1.6} \mathrm{Sb}_{0.4} \mathrm{Te}_{2.94} \mathrm{Se}_{0.06}$ изменяется не более, чем на $2 \%$ по отношению к среднему значению $E_{v}$. В твердом растворе $n$ - $\mathrm{Bi}_{1.8} \mathrm{Sb}_{0.2} \mathrm{Te}_{2.82} \mathrm{Se}_{0.09} \mathrm{~S}_{0.09}$ аналогичные изменения положения $E_{v}$ достигают $6 \%$, что коррелирует с величинами флуктуаций точки Дирака $\Delta E_{\mathrm{D}}$, которые увеличиваются именно в этом составе с высоким фактором мощности и параметром материала (табл. 1).

В отличие от $E_{v}$, положение дна зоны проводимости $E_{c}$ изменяется в пределах $5 \%$ в обоих твердых раствоpax, а величина запрещенной зоны $E_{g}-$ не более, чем 
на 3\% для различных фрагментов поверхности в обоих твердых растворах.

\section{4. Поверхностные уровни дефектов}

Кроме флуктуаций энергии точки Дирака, на нормированных зависимостях дифференциальной туннельной проводимости от напряжения были обнаружены пики, соответствующие поверхностным уровням, образованным дефектами, которые возникают при замещениях атомов в твердых растворах. Энергия дефекта $E_{P}\left(E_{\mathrm{D}}\right)$ определяется относительно энергии точки Дирака $E_{\mathrm{D}}$ и равна $E_{P}\left(E_{\mathrm{D}}\right)=E_{P}-E_{\mathrm{D}}$.

Характерные поверхностные уровни дефектов на двух фрагментах поверхности (0001) (рис. 2, кривые 1,2) наблюдались в составе $n-\mathrm{Bi}_{1.8} \mathrm{Sb}_{0.2} \mathrm{Te}_{2.82} \mathrm{Se}_{0.09} \mathrm{~S}_{0.09}$ с высокими параметром мощности, оптимизированном для комнатной температуры (табл. 1). Интервал энергий уровней 3-9 на первом фрагменте (рис. 2, кривая 1) был наибольшим и $E_{P}\left(E_{\mathrm{D}}\right)=(-118-103.4)$ мэВ при флуктуациях точки Дирака $\Delta E_{\mathrm{D}} /\left\langle E_{\mathrm{D}}\right\rangle=9 \%$, на втором фрагменте (рис. 2, кривая 2) интервал энергий $E_{P}\left(E_{\mathrm{D}}\right)=(-54-15.4)$ мэВ при $\Delta E_{\mathrm{D}} /\left\langle E_{\mathrm{D}}\right\rangle=2.2 \%$. В твердом растворе $n$ - $\mathrm{Bi}_{1.6} \mathrm{Sb}_{0.4} \mathrm{Te}_{2.94} \mathrm{Se}_{0.06}$ с высоким коэффициентом Зеебека поверхностные уровни дефектов в спектрах дифференциальной туннельной проводимости не наблюдались.

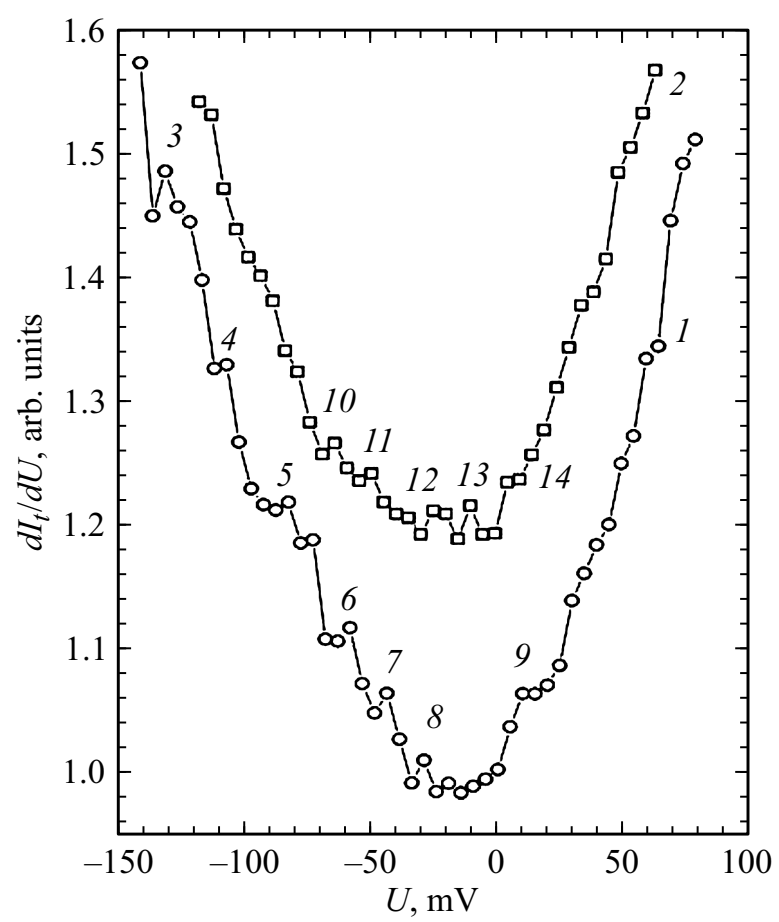

Рис. 2. Нормированная дифференциальная туннельная проводимость $d I_{t} / d U$ в зависимости от напряжения $U(1,2)$ для двух фрагментов на поверхности (0001) твердого раствора $n-\mathrm{Bi}_{1.8} \mathrm{Sb}_{0.2} \mathrm{Te}_{2.82} \mathrm{Se}_{0.09} \mathrm{~S}_{0.09}$. Положения пиков 3-14 соответствуют поверхностным уровням дефектов.
Таблица 2. Энергия точки Дирака $E_{\mathrm{D}}$, волновой вектор $k_{\mathrm{F}}$ и поверхностная концентрация фермионов Дирака $n_{s}$ на поверхности (0001) в твердых растворах $n-\mathrm{Bi}_{1.8} \mathrm{Sb}_{0.2} \mathrm{Te}_{2.82} \mathrm{Se}_{0.09} \mathrm{~S}_{0.09}(1-4)$ и $n-\mathrm{Bi}_{1.6} \mathrm{Sb}_{0.4} \mathrm{Te}_{2.94} \mathrm{Se}_{0.06}(5-7)$

\begin{tabular}{c|l|l|c}
\hline № п. п. & $E_{\mathrm{D}}$, мэВ & $k_{\mathrm{F}}, \mathrm{HM}^{-1}$ & $n_{s} 10^{9} \mathrm{~cm}^{-2}$ \\
\hline 1 & -3.6 & 0.01148 & 0.1 \\
2 & -9.2 & 0.03444 & 0.95 \\
3 & -9.8 & 0.03673 & 1.07 \\
4 & -13.5 & 0.5049 & 2 \\
5 & -66.2 & 0.23554 & 4.41 \\
6 & -93.3 & 0.33196 & 8,77 \\
7 & -99.5 & 0.35438 & 9.99
\end{tabular}

\section{5. Поверхностная концентрация фермионов}

Влияние поверхностных состояний Дирака на термоэлектрические свойства многокомпонентных твердых растворов определяли по величине поверхностной концентрации фермионов $n_{s}$, рассчитанной с учетом флуктуаций энергии точки Дирака в твердых растворах, приведенных на рис. 1. Величину $n_{s}$ рассчитывали как $n_{s}=k^{2} / 4 \pi$, где волновой вектор $k_{\mathrm{F}}=\left|E_{\mathrm{D}}\right| / v_{\mathrm{F}}$, $v_{\mathrm{F}}$ - скорость Ферми, определенная по закону Вегарда из данных [14] для $\mathrm{Bi}_{2} \mathrm{Te}_{3}$, твердых растворов $n-\mathrm{B}_{2} \mathrm{Te}_{3-y} \mathrm{Se}_{y}(y=0.9,1)$ с учетом суммарных замещений атомов в исследованных многокомпонентных твердых растворах. Оценки скорости Ферми показали, что величина $v_{\mathrm{F}}$ возрастает с ростом суммарного количества замещенных атомов в подрешетках $\mathrm{Bi}$ и Те, $v_{\mathrm{F}}=4.25 \cdot 10^{5}$ и $4.05 \cdot 10^{5} \mathrm{M} \cdot \mathrm{c}^{-1}$, соответственно, для $n-\mathrm{Bi}_{1.6} \mathrm{Sb}_{0.4} \mathrm{Te}_{2.94} \mathrm{Se}_{0.06}$ и $n-\mathrm{Bi}_{1.8} \mathrm{Sb}_{0.2} \mathrm{Te}_{2.82} \mathrm{Se}_{0.09} \mathrm{~S}_{0.09}$.

Поверхностная концентрация фермионов $n_{s}$ уменьшается в твердом растворе $n$ - $\mathrm{Bi}_{1.8} \mathrm{Sb}_{0.2} \mathrm{Te}_{2.82} \mathrm{Se}_{0.09} \mathrm{~S}_{0.09}$ (табл. 2) по сравнению с $n$ - $\mathrm{Bi}_{1.6} \mathrm{Sb}_{0.4} \mathrm{Te}_{2.94} \mathrm{Se}_{0.06}$ (табл. 2) на 1-2 порядка в различных точках поверхности (0001) (табл. 2) и уменьшается на 3 порядка по сравнению с твердым раствором $n-\mathrm{B}_{2} \mathrm{Te}_{3-y} \mathrm{Se}_{y}$ [15].

Уменьшение величины $n_{s}$ в $n$ - $\mathrm{Bi}_{1.8} \mathrm{Sb}_{0.2} \mathrm{Te}_{2.82} \mathrm{Se}_{0.09} \mathrm{~S}_{0.09}$ вблизи точки зарядовой нейтральности топологических поверхностных состояний, в которой, по оценкам [10,11], скорость Ферми может увеличиваться в 2-3 раза, обеспечивает рост подвижности фермионов.

Расчеты подвижности $\mu_{0}$, выполненные с учетом эффективного параметра рассеяния $r_{\text {eff }}$, который определяет энергетическую зависимость времени релаксации в виде $\tau=\tau_{0} E^{r}$, где $\tau_{0}-$ константа, не зависящая от энергии, показали, что $\mu_{0}$ возрастает в составе с замещениями атомов $\mathrm{Te} \rightarrow \mathrm{Se}+\mathrm{S}$ [9] (табл. 1).

Таким образом, исследования спектров дифференциальной туннельной проводимости в многокомпонентных твердых растворах показали, что вклад поверхностных состояний возрастает в составе $n-\mathrm{Bi}_{1.8} \mathrm{Sb}_{0.2} \mathrm{Te}_{2.82} \mathrm{Se}_{0.09} \mathrm{~S}_{0.09}$ с высоким параметром мощ- 
ности за счет значительного уменьшения поверхностной концентрации $n_{s}$ вблизи точки зарядовой нейтральности и роста подвижности фермионов по сравнению с $n-\mathrm{Bi}_{1.6} \mathrm{Sb}_{0.4} \mathrm{Te}_{2.94} \mathrm{Se}_{0.06}$.

\section{6. Заключение}

Проведены исследования дифференциальной туннельной проводимости межслоевой поверхности Ван-дер-Ваальса (0001) в твердых растворах $n-\mathrm{Bi}_{1.8} \mathrm{Sb}_{0.2} \mathrm{Te}_{2.82} \mathrm{Se}_{0.09} \mathrm{~S}_{0.09}$ с высоким фактором мощности и оптимальными термоэлектрическими свойствами для температур, близких к комнатной, и в $n-\mathrm{Bi}_{1.6} \mathrm{Sb}_{0.4} \mathrm{Te}_{2.94} \mathrm{Se}_{0.06}$ с высоким коэффициентом Зеебека и оптимальными термоэлектрическими свойствами для низких температур.

Флуктуации энергии точки Дирака $\Delta E_{\mathrm{D}}$ и смещение потолка валентной зоны $\Delta E_{\mathrm{V}}$ от среднего значения, определенные для различных фрагментов поверхности в твердом растворе $n$ - $\mathrm{Bi}_{1.8} \mathrm{Sb}_{0.2} \mathrm{Te}_{2.82} \mathrm{Se}_{0.09} \mathrm{~S}_{0.09}$ возрастают по сравнению с составом $n-\mathrm{Bi}_{1.6} \mathrm{Sb}_{0.4} \mathrm{Te}_{2.94} \mathrm{Se}_{0.06}$. В составе $n-\mathrm{Bi}_{1.8} \mathrm{Sb}_{0.2} \mathrm{Te}_{2.82} \mathrm{Se}_{0.09} \mathrm{~S}_{0.09}$ с высокими флуктуациями $\Delta E_{\mathrm{D}}$ в запрещенной зоне наблюдаются поверхностные уровни, образованные примесными дефектами.

В твердом растворе $n$ - $\mathrm{Bi}_{1.8} \mathrm{Sb}_{0.2} \mathrm{Te}_{2.82} \mathrm{Se}_{0.09} \mathrm{~S}_{0.09}$ с высоким параметром мощности точка Дирака $E_{\mathrm{D}}$ находится в окрестности точки зарядовой нейтральности топологических поверхностных фермионов, обладающих более высокой скоростью и подвижностью. При этом поверхностная концентрация фермионов $n_{s}$ становится на 2 порядка меньше, чем в составе $n-\mathrm{Bi}_{1.6} \mathrm{Sb}_{0.4} \mathrm{Te}_{2.94} \mathrm{Se}_{0.06}$.

\section{Конфликт интересов}

Авторы заявляют, что у них нет конфликта интересов

\section{Список литературы}

[1] M.Z. Hasan, C.L.Kane. Rev. Mod. Phys., 82, 3045 (2010).

[2] A. Parbatani, E.S. Song, J. Claypoole, B.Yu. Nanotechnology, 30, 165201 (2019).

[3] J. Gooth, B. Hamdou, A. Dorn, R. Zierold, K. Nielsch. Appl. Phys. Lett., 104, 243115 (2014).

[4] R. Dey, T. Pramanik, A. Roy, A. Rai, S. Guchhait, S. Sonde, H.C.P. Movva, L. Colombo, L.F. Register, S.K. Banerjee. Appl. Phys. Lett., 104, 223111 (2014).

[5] G. Jiang, J. Yi, L. Miao, P. Tang, H. Huang, C. Zhao, S. Wen. Sci. Rep., 8, 2355 (2018).

[6] G. Zhang, B. Kirk, L.A. Jauregui, H. Yang, X. Xu, Y.P. Chen, Y. Wu. Nano Lett., 12, 56-60 (2012).

[7] Y. Hou, R. Wang, R. Xiao, L. McClintock, H.C. Travaglini, J.P. Francia, H. Fetsch, O. Erten, S.Y. Savrasov, B. Wang, A. Rossi, I. Vishik, E. Rotenberg, D. Yu. Nature Commun., 10, 5723 (2019).

[8] H. Nam, Y. Xu, I. Miotkowski, J. Tian, Y.P. Chen, C. Liu, C.K. Shih. J. Phys. Chem. Solids, 128, 251 (2019).

[9] L.N. Lukyanova, V.A. Kutasov, P.P. Konstantinov, V.V. Popov. 2012 Optimization of solid solutions based on bismuth and antimony chalcogenides above room temperature Modules,Systems, and Applications in Thermoelectrics, ed. by D.M. Rowe (Boca Raton, FL: CRC Press) pp 7-1-718.

[10] M. Hirata, A. Kobayashi, C. Berthier, K. Kanoda. Rep. Progr. Phys., 84, 036502 (2021).

[11] L. Zhao, M. Konczykowski, H. Deng, I. Korzhovska, M. Begliarbekov, Z. Chen, E. Papalazarou, M. Marsi, L. Perfetti, A. Hruban, A. Wołos, L. Krusin-Elbaum. Nature Commun. 7, 10957 (2016).

[12] L.N. Lukyanova, I.V. Makarenko, O.A. Usov. J. Phys.: Condens. Matter, 32, 465701 (2020).

[13] C. Wagner, R. Franke, T. Fritz. Phys. Rev. B, 75, 235432 (2007).

[14] H. Liu, S. Liu, Ya. Yi, H. He, J. Wang. 2D Mater., 2, 045002 (2015).

[15] Л.Н. Лукьянова, И.В. Макаренко, О.А. Усов, П.А. Дементьев. ФТП, 53, 654 (2019).

\section{Differential tunneling conductance in multicomponent solid solutions $\mathbf{B i}_{2-x} \mathbf{S b}_{x} \mathbf{T e}_{3-y-z} \mathbf{S e}_{y} \mathbf{S}_{z}$ \\ L.N. Lukyanova, I.V. Makarenko, O.A. Usov \\ loffe Institute, 194021 St. Petersburg, Russia}

Abstract Differential tunnel conductance of the interlayer van der Waals surface (0001) was investigated in $n-\mathrm{Bi}_{2-x} \mathrm{Sb}_{x} \mathrm{Te}_{3-y-z} \mathrm{Se}_{y} \mathrm{~S}_{z}$ multicomponent solid solutions at $x=0.2, y=z=0.09$, optimized for near room temperatures with a high power factor, and at $x=0.4, y=0, z=0.06$ with optimal thermoelectric properties for low temperatures and a high Seebek coefficient. It is shown that the intensity of the fluctuations of the Dirac point energy $\Delta E_{\mathrm{D}}$, a shift the top of the valence band and presence of surface levels in the energy gap formed by impurity defects is determined with composition and thermoelectric properties of solid solutions. The contribution of the surface states of Dirac fermions increases in $n$ - $\mathrm{Bi}_{1.8} \mathrm{Sb}_{0.2} \mathrm{Te}_{2.82} \mathrm{Se}_{0.09} \mathrm{~S}_{0.09}$ solid solution with a high power factor due to significant decrease of the surface concentration near the charge neutrality point and the growth of fermion mobility. 